\title{
Post Tonsillectomy Haemorrhage In Bipolar Diathermy Versus Conventional Technique
}

\author{
Esam-eddin Bakheit Mohammed Ahmed ${ }^{1}$, Faroug Bakheit Mohammed Ahmed ${ }^{2}$ \\ ${ }^{1}$ ENT Department, Faculty of Medicine, Shendi University, Shendi, Sudan \\ ${ }^{2}$ Faculty of Science and Technology, Shendi University, Shendi, Sudan
}

Email address:

asimelsonni@hotmail.com (Esam-eddin B. M. A.)

\section{To cite this article:}

Esam-eddin Bakheit Mohammed Ahmed, Faroug Bakheit Mohammed Ahmed. Post Tonsillectomy Haemorrhage In Bipolar Diathermy Versus Conventional Technique. Clinical Medicine Research. Vol. 4, No. 6, 2015, pp. 189-194. doi: 10.11648/j.cmr.20150406.14

\begin{abstract}
This is a prospective hospital based study conducted in Omdurman Military Hospital (OMH) from January to December 2009 comparing the conventional dissection tonsillectomy (CDT) versus bipolar diathermy tonsillectomy (BDT). The study was conducted to determine the incidence of post-tonsillectomy haemorrhage and it's relation to technique used. Also to detect the efficacy of bipolar diathermy in intra-operative haemostasis in tonsillectomy procedures. 165 patients who had tonsillectomy during the study period were randomly collected and grouped into two according to the technique used. Questionnaire and SPSS program were used for data collection and analysis respectively. The primary haemorrhage was reported in $3.6 \%$ and the secondary one in $1.8 \%$ of study sample. The intra-operative bleeding found to be correlated (p $<0.005)$ to the technique. Insignificant secondary haemorrhage occurred in three cases from the bipolar diathermy group but had no correlation $(p>0.005)$ with the technique used for tonsillectomy. The primary haemorrhage was found to be correlated to the technique used. The bipolar diathermy is highly efficacious in intra-operative haemostasis during tonsillectomy. The difference was insignificant between bipolar diathermy and conventional dissection methods regarding the secondary haemorrhage.
\end{abstract}

Keywords: Tonsillectomy, Bipolar, Diathermy and Conventional

\section{Introduction}

\subsection{Anatomy of Tonsils}

More detailed anatomical and histological studies were done in the 19th century, mainly the ones by Wilhelm Von Waldeyer, who described lymphatic tissues in the pharyngeal area $[1,2]$. The palatine tonsil is a mass of lymphoid tissue situated in the lateral wall of the oropharynx. This ring surrounds the openings into the digestive and respiratory tracts and consists of the palatine and tubal tonsils laterally, the nasopharyngeal tonsil (adenoids) and smaller collections of lymphoid tissue in the intertonsillar intervals posterosuperiorly, and the lingual tonsils anteroinferiorly. The medial (free) surface of the palatine tonsil projects into the oropharynx and usually presents a pitted appearance. These pits, 10-15 in number, lead into a system of blind-ending, often highly branching crypts, which extend through the whole thickness of the tonsil. The deep (lateral) aspect of the tonsil is covered by the fibrous tissue of the tonsillar hemicapsule that can be easily separated throughout most of its extent from the underlying superior constrictor muscle. An important and sometimes large vein (the external palatine or paratonsillar vein) descends from the soft palate lateral to the tonsillar hemicapsule before piercing the pharyngeal wall. Haemorrhage from this vessel can complicate tonsillectomy. The size of the tonsil varies greatly and at puberty may be approximately $10-15 \mathrm{~mm}$ in transverse diameter and approximately $20-25 \mathrm{~mm}$ in the vertical dimension[3].

\subsection{Physiology of Tonsils}

The lymphoid tissue of Waldeyer's tonsillar ring contains B-cell lymphocytes, T-cell lymphocytes, and a few mature plasma cells. This tissue is primarily involved in inducing secretory immunity and regulating immunoglobulins production. The location of Waldeyer's tonsillar ring and its design allow direct exposure of the immunologically active cells to foreign antigens entering the upper aerodigestive tract, which maximizes the development of immunologic memory. These tissues are most active from the ages of 4 to 10 and tend to involute after puberty. After their involution, the secretory immune function of these tissues remains, but not at the same level as previously [4]. 


\subsection{Historical Background for Tonsillectomy}

Cornélio Celsus, in the first century B.C., was the first to describe tonsillectomy procedure. He reported the procedure performance for dissection and removal of the structures. Celsus applied a mixture of vinegar and milk in the surgical specimen for hemostasis and also described his difficulty doing that due to lack of proper anesthesia. Joseph Beck was the first one to describe the use of a device with cutting wire inside a rigid ring known as Beck-Mueller's ring. An instrument that also gained publicity in that period was Sluder's guillotine. At the beginning of 20th century, the use of forceps and scalpels resulted in less bleeding. From 1909, tonsillectomy surgery became a common and safe procedure, when Cohen adopted ligature of bleeding vessels to control per-operative hemorrhage. Sluder's tonsilotome had its first routinely uses in the United States in 1909, in Austria in 1910, and in Belgium in 1912 [1,2].

Tonsillectomy techniques are currently undergoing something of revolution. Until about few years ago dissection tonsillectomy (first described by Edwin Pynchon in 1890), with haemostasis performed with ties or diathermy was the standard but more recently there has been an explosion of different dissection instruments described in an effort to try and reduce postoperative pain and haemorrhage associated with this procedure. Dissection techniques include: cold dissection (sharp / blunt), diathermy or electrocautery (monopolar / bipolar forceps / bipolar cissor) and many others. These various techniques have been developed in attempt to minimize tissue trauma and thereby postoperative pain and bleeding while remaining simple and of short duration. All techniques have their advocates and detractors, and some comparisons between techniques have been made ${ }^{[3]}$. Electrocautery refers to using a heated instrument to cut or coagulate tissues. Diathermy is quicker for haemostasis but possibly related to increased risk of secondary haemorrhage. It may be the method of choice in patients at high risk of bacteraemia ${ }^{[5]}$. The use of diathermy to secure haemostasis in tonsillectomy remains controversial in U. K. (Murty \&Watson, 1990 ), where $56 \%$ of otolaryngologists do not use diathermy, thinking it to increase postoperative haemorrhage rates and patient morbidity ${ }^{[6]}$. With unipolar diathermy it is difficult to control depth of heat coagulation and subsequent devitalization since this will depend on the power released at the site of application and a small variation in tissue depth in tonsillar fossa can involve adjacent vital structures resulting in variable postoperative pain. With bipolar diathermy, area of tissue coagulation is localized between the fine tips of diathermy forceps causing less tissue damage in a more controlled and precise fashion resulting in less variable postoperative pain. Ligations do not cause deep seated tissue damage but they may catch muscle fibres on the floor of tonsillar fossa resulting in more post operative pain [7].

\subsection{Complications of Tonsillectomy}

Haemorrhage is one of the most common complications. The reactionary haemorrhage (bleeding post-operatively and within the first 24 hours) is the most feared complication because of the risk of airway obstruction, shock and ultimately death, if inappropriately treated or untreated. Need to return to theatre reported in $1 \%$ of the overall variable incidence. They usually happen in the first eight hours after surgery and majority within the first four hours. Various classification systems for the severity of bleeding have been devised. All are subjective and grade haemorrhage into minor and major with further subdivision of the latter into those that do or do not require return to theatre and/or require transfusion. None of the operative techniques seems to be significantly more prone to reactionary haemorrhage than the others. Likewise it's incidence is not related to grade and seniority of the surgeon ${ }^{[8]}$. A review on secondary haemorrhage suggests that it may be more common than widely appreciated, occurring in about $9 \%$ of cases. Of these, $1.4 \%$ were severe requiring a return to theatre. Most (70\%) present between days four and seven. A retrospective study of 15,218 showed a $0.4 \%$ secondary haemorrhage rate [9].

\subsection{Studies Related to Our Topic}

All of the studies found in literature regarding our topic were international and no comparative study on bipolar diathermy tonsillectomy found to be conducted in Sudan.

Barr (2002), conducted study on how to reduce diathermy in tonsillectomy, to minimize excessive tissue burn and in turn post-operative pain. He found no cases of primary hemorrhage had occurred (defined as occurring in the first 24 hours). Significant secondary hemorrhage classified as moderate was found in seven patients $(2.1 \%)$. Only four patients in total $(1.2 \%)$ required to return to the theatre. One of these patients $(0.3 \%)$ needed transfusion and being classified as severe hemorrhage. All other patients settled with intravenous antibiotic treatment for secondary infection. Eight patients had minor or insignificant hemorrhage, either spitting a clot of blood or some blood stained saliva and had no active bleeding when seen. Including these patients he found that the overall secondary hemorrhage rate was $4.8 \%$ and occurred between 1 and 11 days post-operatively. Two adult patients ( 0.6 per cent) were readmitted for control of pain, one patient for 1 day and the other for 2 days [10].

Malik et al (1982), conducted a trial in India comparing ligation to diathermy (spot or zonal) in 450 cases, they found no significant difference in haemorrhage rates but diathermy was faster than ligation ${ }^{[11]}$.

Roberts et al (1982), studied 1090 cases prospectively and found slight excess of primary haemorrhage associated with ligation and slight excess of secondary haemorrhage with diathermy, but the difference was not statistically significant [12].

K. Haddow (2004), designated a prospective, randomized, controlled trial. One hundred and forty-one patients ( 58 per cent) were randomized to the bipolar dissection and 104 (42 per cent) to the cold dissection groups. Seventeen patients (12.1 per cent) in the bipolar dissection group and eight patients (7.7 per cent) in the cold dissection group suffered haemorrhage $(\mathrm{p}=1.0)$. The haemorrhage rates for procedures 
conducted by senior house officers, specialist registrars and consultants were 11.4 per cent, 10.3 per cent and 5.0 per cent, respectively. Two patients required surgical intervention, both from the bipolar dissection group. No patients required blood transfusion. A history of quinsy was not associated with an increased haemorrhage rate. He concluded that the difference in haemorrhage rates between groups and surgeon grades did not reach statistical significance. Nonetheless the trend towards a greater incidence of haemorrhage in the bipolar group and in procedures conducted by more junior surgeons during the trial raised concerns. The results of the National Prospective Tonsillectomy Audit and their interim results have led them to abandon the trial and disallow the use of bipolar dissection in tonsillectomies performed by junior staff members [13].

Y. T. Pang (1995), designed prospective study to evaluate the electrodissection technique against the conventional dissection/snare technique. He introduced sixty children into each section of the study (total 120 children). He found a statistically significant shorter operating time (mean 11.2 minutes) and lower intraoperative blood loss (mean $5 \mathrm{ml}$ ) using the bipolar diathermy technique. Post-operatively, the children who had bipolar diathermy tonsillectomy were found to be able to drink and eat significantly earlier than the cold dissection group. He detected no measurable difference in analgesia requirements before discharge and the time of discharge between the two methods. Post-operative haemorrhage occurred in two out of 60 (3.4 per cent) and one out of 60 (1.7 per cent children) in the conventional and bipolar diathermy dissection, respectively, but this was not statistically significant [14].

This study is aim to

1. Determination of incidence of post tonsillectomy haemorrhage (intra- operative and secondary).

2. Detection of efficacy of diathermy in intra-operative haemostasis during tonsillectomy.

3. Identification of the relation, if any, between post tonsillectomy haemorrhage and operative technique used.

4. Post operative satisfaction of the patient and/or his/her family.

\section{Methods and Materials}

\subsection{Methods}

Bipolar diathermy and cold dissection instruments were used for performing the procedures and statistical packages for social sciences (SPSS) program for data analysis.

\subsection{Materials}

Patients who have had tonsillectomy in Omdurman Military Hospital (OMH) during the study period (January to December 2009).

\subsection{Exclusion Criterion}

Operations done by cold dissection technique and in which bipolar diathermy used for haemostasis.

\section{Results}

A sample of 165 patients who have had tonsillectomy in $\mathrm{OMH}$ during the study period was randomly selected including those operated by both CDT and BDT techniques. In BDT group the bipolar diathermy was used during the whole procedure for dissection and haemostasis. Patients from KTH planned to be incorporated but none had been operated using the bipolar diathermy technique during the study period, so were omitted for unrepresentative sample. According to severity the intra-operative blood loss was classified into severe $(>250 \mathrm{ml})$, moderate $(100-250 \mathrm{ml})$, mild $(25-100 \mathrm{ml})$ and no "insignificant" $(<25 \mathrm{ml})$ bleeding. The loss was estimated depending on amount in suction bottle and number of gauze pieces used (10ml for each fully soaked large piece). In bipolar diathermy operations usually very small gauze pieces were used to clear the field prior to cauterization of the bleeding vessels, whereas in those performed conventionally large (complete) pieces needed for compression to aid in haemostasis. The severe intra-operative bleeding was considered as primary haemorrhage.

The age group $(5-10)$ years was the largest $(32.1 \%)$ as evident in table (1). The sex distribution shown in table (2) and majority $(63 \%)$ were males. The size of tonsils on pre-operative examination was graded on oropharyngeal obstruction into four grades. Grade I 25\%, grade II 50\%, grade III $75 \%$ and grade IV $100 \%$ (tonsils touch each other) obstruction. The study sample was grouped into three based on tonsillar size with grade II and III in one group for the subjectivity of the grading system and this was shown in table (3).

The primary haemorrhage (bleeding which occurred in the first 24 hours peroperatively) including in our study the severe intra-operative bleeding was reported in $3.6 \%$ of cases and all of them from those operated by CDT (6.3\% of this group) as evident from figure (1). This figure again showed that not only the other degrees of bleeding were higher in CDT than BDT, but also all cases regarded to have no bleeding were operated with bipolar technique. No reactionary haemorrhage observed in the study sample.

The secondary haemorrhage (bleeding which occurred after 24 hours of operation) was reported in $1.8 \%$ (3 cases) of the sample. All these cases found to be operated by bipolar technique and account for $4.3 \%$ of this group as explained in figure (2). All patients showed up again after seven days and only $11.6 \%$ in CDT and $12.8 \%$ in BDT suffered minor complaints (figure 4) including; referred otalgia, odynophagia and spitting of blood.

Table (4) showed that there is very strong positive correlation $(p=0.00)$ between the technique used and occurrence and severity of intra-operative bleeding which was more evident and more severe in CDT. From table (5) it is clear that there is no correlation $(p>0.005)$ between the incidence of secondary haemorrhage and operative technique. Despite that the reported three cases were from BDT group there was no significant difference $(p>0.05)$ between the two techniques. The tonsillar size on examination showed no 
correlation $(\mathrm{p}>0.005)$ with the secondary haemorrhage in BDT (table 6$)$, but a negative one $(\mathrm{p}=0.00)$ with the degree of intra-operative bleeding in CDT (table 7).

Table 1. Age distribution of the study sample.

\begin{tabular}{lll}
\hline Age group & NO. & \% \\
\hline$<5$ & 47 & 28.5 \\
$5-10$ & 53 & 32.1 \\
$11-20$ & 41 & 24.9 \\
$>20$ & 24 & 14.5 \\
Total & 165 & 100 \\
\hline
\end{tabular}

Table 2. Sex distribution of the study sample.

\begin{tabular}{lll}
\hline Sex & NO. & \% \\
\hline Male & 104 & 63 \\
Female & 61 & 37 \\
Total & 165 & 100 \\
\hline
\end{tabular}

Table 3. Size of tonsils on pre-operative examination of study sample.

\begin{tabular}{lll}
\hline Size & NO. & \% \\
\hline Grade I & 14 & 8.5 \\
Grade II/III & 29 & 17.6 \\
Grade IV & 122 & 73.9 \\
Total & 165 & 100 \\
\hline
\end{tabular}

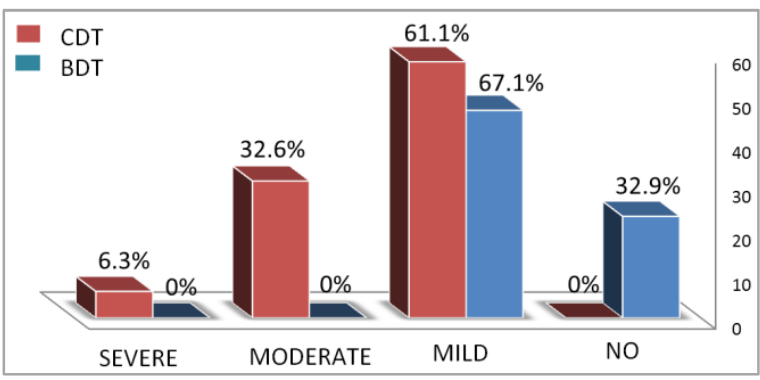

Figure 1. Incidence of primary haemorrhage in both techniques.

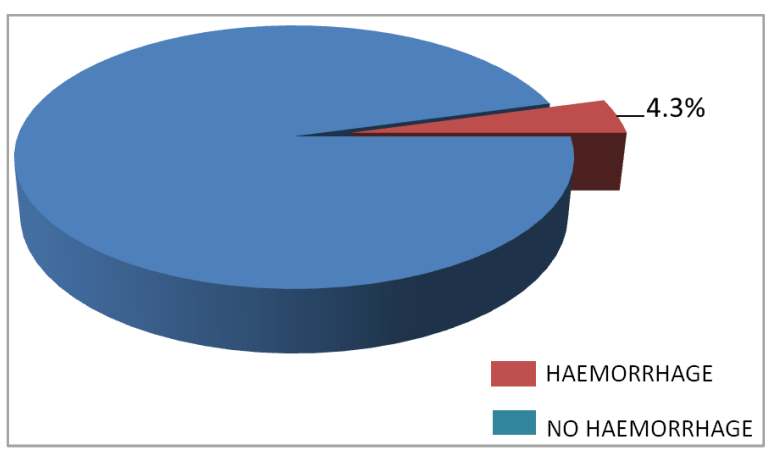

Figure 2. Incidence of secondary haemorrhage in bipolar technique.

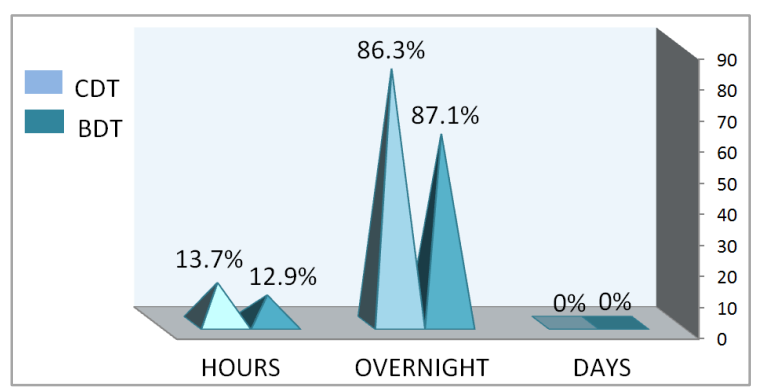

Figure 3. Post-tonsillectomy stay in hospital in both techniques.

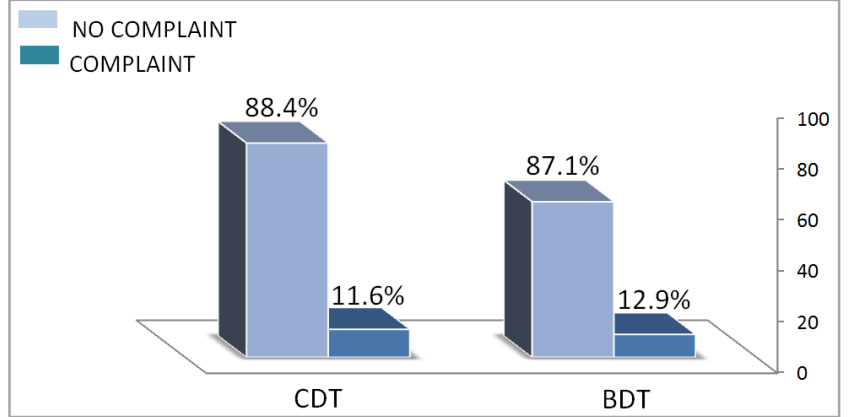

Figure 4. Incidence of post-tonsillectomy first follow up visit complaints.

Table 4. Correlation between intra-operative bleeding and tonsillectomy technique.

\begin{tabular}{llll}
\hline Bleeding & BDT & CDT & Total \\
\hline No & 23 & 0 & 23 \\
Mild & 47 & 58 & 105 \\
Moderate & 0 & 31 & 31 \\
Severe & 0 & 6 & 6 \\
Total & 70 & 95 & 165 \\
\hline
\end{tabular}

$\mathrm{P}=0.00$

Table 5. Correlation between secondary haemorrhage and tonsillectomy technique.

\begin{tabular}{llll}
\hline Technique & BDT & CDT & Ttal \\
Haemorrhage & & 0 & 3 \\
Yes & 3 & 95 & 162 \\
No & 67 & 95 & 165 \\
Total & 70 & & \\
\hline
\end{tabular}

$\mathrm{P}=0.42$

Table 6. Correlation between secondary haemorrhage and size of tonsils in diathermy group.

\begin{tabular}{|c|c|c|c|c|}
\hline Size & Haemorrhage & Yes & No & Total \\
\hline Grade I & & 1 & 10 & 11 \\
\hline Grade II/III & & - & 20 & 20 \\
\hline Grade IV & & 2 & 37 & 39 \\
\hline Total & & 3 & 67 & 70 \\
\hline
\end{tabular}

$\mathrm{P}=0.38$

Table 7. Correlation between intra-operative bleeding and size of tonsils in conventional tonsillectomy group.

\begin{tabular}{|c|c|c|c|c|}
\hline Size $\quad$ Bleeding & Mild & Moderate & Severe & Total \\
\hline Grade I & 5 & 1 & - & 6 \\
\hline Grade II/III & 5 & - & 6 & 11 \\
\hline Grade IV & 48 & 30 & - & 78 \\
\hline Total & 58 & 31 & 6 & 95 \\
\hline
\end{tabular}

$\mathrm{P}=0.00$

\section{Discussion}

Our study detected that the incidence of primary haemorrhage was $3.6 \%$ of the overall sample; all of them were operated conventionally, and account for $6.3 \%$ of CDT group (figure 1). This result is similar to that by Barr who found no primary haemorrhage in patients operated by diathermy. Our 
result is also better than that by $\mathrm{K}$. Haddow et al, which was $12.1 \%$ for overall incidence of haemorrhage in diathermy. It also detected that the other degrees of bleeding were higher in CDT group and even all those regarded to have no bleeding ( $<$ $25 \mathrm{ml}$ ) were operated with bipolar technique. This indicates that bipolar diathermy is highly efficient in post tonsillectomy haemostasis with the least intra-operative blood loss compared to conventional method. This result is correspondent to that reported by Pang who found a lower intra-operative blood loss (mean $5 \mathrm{ml}$ ) using bipolar diathermy.

All cases $(1.8 \%)$ of secondary haemorrhage (bleeding which occurred after 24 hours of operation) found to be operated by bipolar technique and account for $4.3 \%$ of this group as explained in figure (2). All of them had no severe haemorrhage, but only a history of minor bleeds in form of spitting of minimal blood. This history was reported to occur on days five to seven post-operatively and amount of loss was considered insignificant as it neither disturb the patient nor mandate a return not to the theatre, but even immediately back to the hospital. On first post-operative visit examination these patients had no active nor evidence of bleeding and treated conservatively with no need for hospital admission. These results are better when compared to those by Barr who reported a $4.8 \%$ incidence of secondary haemorrhage with the use of diathermy, of them $1.2 \%$ required return to theatre and $0.3 \%$ required transfusion. Our results are in favour of conventional technique when compared to those of Pang who reported secondary haemorrhage in $1.7 \%$ of diathermy and in $3.4 \%$ of cold dissection group. Regarding the time of secondary haemorrhage our results are not different from that in literature by Alexander et al ( $5-7$ days post-operatively) but disagree with the wide range ( 1 - 11 days post-operatively) observed by Barr.

Despite that the reported three cases of secondary haemorrhage were from BDT group there was no significant difference $(p>0.05)$ between the two techniques (table 5). As mentioned earlier these patients suffered minor bleeds in form of spitting of blood on days five to seven after the operation. This can be attributed to minor oozes from larger vessels with contracture of healing fibrous tissue, as these vessels usually ligated in conventional technique and no other explanation as infection noted.

The positive very strong correlation $(p=0.00)$ between the technique used and occurrence and severity of intra-operative bleeding which was more evident and more severe in CDT (table 4) indicates that the amount of intra-operative blood loss may be technique dependent as the primary haemorrhage was not reported in BDT. However, it is worth to mention that all diathermy operations were performed by surgeon while all conventional operations by registrars, so the skill factor might be implicated in this and need to be freed.

According to Malik et al, and Watson et al, there was no significant difference in incidence of haemorrhage in both techniques. Our result is similar to this in respect of secondary haemorrhage $(p>0.05)$, but dissimilar regarding the primary haemorrhage where the difference between the two techniques is significant $(p<0.05)$. Also dissimilar to Pang results where there was no significant difference regarding the secondary haemorrhage.

Our results are similar to the findings of Roberts et al, where there was slight excess of primary haemorrage in conventional technique and slight excess of secondary in diathermy group. But disagree with their results in difference between the two techniques which was significant $(p<0.05)$ for the primary haemorrhage in our study and insignificants in theirs for both types of haemorrhage.

\section{Conclusions and Recommendations}

The incidence of primary (severe intra-operative) haemorrhage was $3.6 \%$ and related to tonsillectomy by conventional dissection technique. The bipolar diathermy is highly efficacious for intra-operative haemostasis during tonsillectomy. There is correlation between post tonsillectomy primary haemorrhage and the operative technique with which the procedure performed. No significant secondary haemorrhage reported, but minor bleeds in form of spitting of blood in $1.8 \%$ of the study sample on day five to seven post-operatively and in regard to this, the difference was insignificant between bipolar diathermy and conventional methods.

We recommend promotion of bipolar diathermy usage in performing tonsillectomy wherever possible. Application of bipolar diathermy tonsillectomy in training program of otolaryngology specialty. Further local comparative studies to reinforce or disagree with our results. Freeing of the effects of variability in levels of surgeons in after-coming studies to produce more reliable technical effect on post-operative complications.

\section{References}

[1] Young J. R, Bennett J. History of Tonsillectomy. ENT News (2004), 13:34-35.

[2] Feldmann H., 200 year history of tonsillectomy. Images from the history of otorhinolaryngology, highlighted by instruments from the collection of the German Medical History Museum in Ingolstadt. Laryngorhinootologie (1997), 76(12): 751-60.

[3] Scott-Brown's Otorhinolaryngology, Head and Neck Surgery, Tonsillectomy techniques $7^{\text {th }}$ edition (2008), 2:1991.

[4] Nave H, Gebert A, Pabst R. Morphology and immunology of the human palatine tonsil. Anat Embryol. (2001), 204:367. (Review summarizing current data on anatomy, histology, pathology, and immunology of palatine tonsils.) [PMID: 11789984].

[5] Kocturks S, Yildirim A, Demiary T, Bahar G, Bakici MZ. Cold dissection versus bipolar cauterizing tonsillectomy for bacteraemia. American Journal of otolaryngology (2005), 26:51-3.

[6] P.K.Moonka- Ligation Vs. Bipolar Diathermy for Haemostasis in Tonsillectomy - A Comparative Study Indian Journal of Otolaryngology and Head and Neck Surgery Vol. 54 No. I, January-March 2002 
[7] Choy, A. T. K. el al: Journal of laryngology \& otology (1992), 106: $21-22$.

[8] Kendrich D, Gibbin K. An audit of the complications of paediatric tonsillectomy, adenoidectomy and adenotonsillectomy. Clinical otolaryngology (1993), 18(2): $115-7$.

[9] Alexander RJ, Kukreja R, Ford GR. Secondary post tonsillectomy haemorrhage and informed consent. Journal of laryngology and otology (2004), 118: 937-940.

[10] G. D. Barr. How To Reduce Diathermy In Tonsillectomy Vessel Dissection Tonsillectomy (VDT): Description Of Technique And Analysis Of Results. The Internet Journal of Otorhinolaryngology (2006), Volume 4 Number 2.
[11] Malik, M. K.. Bhatia. B. P. R. Kumar. A. Control of haemorrhage in tonsillectomy. Journal of Indian Medical Association (1992), 79: 115-117.

[12] Roberts, C, Jayaramchandran, S., Raine, C. H. A prospectiave study of factors which may predispose to postoperative tonsillar fossa haemorrhage. Clinical Otolaryngology (1992), 17: 13-17.

[13] K Haddow, M. L. Montague and S. M. Hussain. The Journal of Laryngology \& Otology (2006), 120: 450-454.

[14] Y. T. Pang: Paediatric tonsillectomy: bipolar electrodissection and dissection/snare compared. Department of Otolaryngology, University of Manchester School of Medicine, Hope Hospital, Salford, UK. The Journal of Laryngology \& Otology (1995), 109:733-736. 